\title{
The two meanings of a homophone*
}

\author{
BENNY SHANON
}

\author{
Massachusetts Institute of Technology, Cambridge, Massachusetts 02139
}

\begin{abstract}
Ss were presented with sentences whose main noun was either a homophone or not. Following each sentence, Ss were presented with a single noun and they had to indicate whether it was relevant to the sentence or not. More specifically, irrelevant probes following sentences containing homophones were either irrelevant with respect to both interpretations of the homophone or irrelevant with respect to the present interpretation of the homophone but relevant with respect to its other interpretation. The data suggest that people compare the main noun and the probe, and that in doing so they compute both entries of the homophone noun. No effect was found due to a variation in the delay between the presentation of the sentence and the presentation of the probe.
\end{abstract}

This paper examines how people process lexical anbiguities, i.e., homophones. In general, one can distinguish between two models for the processing of ambiguous linguistic items. By the first model, people process only one possible interpretation at a time; if this interpretation is found to be inappropriate, they process the other interpretation (cf. Lashley, 1951). In other words, the model suggests that ambiguous linguistic items are treated like unambiguous ones. Carey, Mehler, and Bever (1970) called this suggestion the unitary perception hypothesis. Alternatively, the exhaustive computation hypothesis (cf. Carey et al, 1970) suggests that people always compute all possible interpretations.

In the following paragraphs, I summarize some of the data supporting the two hypotheses. Although most of the studies address the problem of structural ambiguity, I believe the distinction made by the two candidate hypotheses reflects basic strategies for the processing of a mbiguity in general.

First, consider the data supporting the unitary perception hypothesis. Carey et al (1970) set Ss to receive an unambiguous adjectival (e.g., "They are sleeping lions") or progressive (e.g., "They are discussing books") sentence and in fact presented them with an ambiguous sentence (e.g., "They are lecturing doctors"). The sentence was followed by a picture which made it true or false on one of its readings. The verification times collected from Ss who responded to the sense compatible with the set were shorter than those collected from Ss who responded to the incompatible sense. Furthermore, the former verification times were not different from those collected from Ss who responded to analogous unambiguous sentences.

*This research was supported in part by United States Public Health Service Research Grant MH 20021 from the National Institute of Mental Health. I am indebted to Herbert $\mathrm{H}$. Clark and Lucia Kellar for their helpful comments.
Other investigators argue for the exhaustive computation hypothesis. McKay (1966) demonstrated that the time required to complete an ambiguous sentence fragment is longer than the time required to complete a similar unambiguous fragment. The effect was found with both lexical and structural ambiguities, and was largest with deep structure ambiguities. Fodor and Garrett (1967) pointed out that verbs taking various kinds of complements are psychologically more difticult than verbs taking only one kind of complement. They attributed this effect to an exhaustive scan of possible deep structure configurations. Fodor, Bever, and Garrett (1968) have interpreted in a similar way the relative complexity of verbs having several underlying structures. Lackner and Garrett (1972) found that the interpretation of an ambiguous sentence is affected by a simultaneously presented disambiguating context. However, Ss could not report any information concerning the content of the unattended disambiguous sentence. The authors concluded that both interpretations of an ambiguous sentence are immediately available during its processing. It will be noted that the same pattern of results appeared with structural and lexical ambiguities.

Garrett (1970) and Bever, Garrett, and Hurtig (1973) suggested that the two hypotheses are not mutually exclusive but, rather, reflect various stages in the linguistic processing. They pointed out that experiments which show an effect of ambiguity used tasks initiated during the processing of the sentence in question, while those showing no such effect used tasks initiated after processing of the sentence had been completed. Bever et al (1973) found longer reaction times prior to clause closure than following it. They interpreted this as indicating that when presented with an ambiguous clause Ss process both interpretations, but immediately after the clause is over they recode it with only one meaning retained. 
The effect was significant only with deep structure ambiguities, but similar trends appeared with lexical and surface siructure ambiguities as well.

In this paper, I report two experiments, which, except for one parameter, were identical. In both experiments, $S$ s were presented with sentences whose main noun may have been a homophone. For example, in (1) the main noun is a homophone; in (2) the main noun is not a homophone.

(1) The organ is healthy.

(2) The teacher is strict.

Following the presentation of the sentence, the Ss were presented with a single word and they were asked to indicate whether the probe was relevant to the sentence or not. Thus, body is relevant to (1), but music and zoo are not.

Basically, there are two strategies that Ss may use in order to execute the task in question. First, they may process the sentence, retain its interpretation, and then, when the probe is presented to them, decide whether it is relevant to the sentence interpretation or not. Secondly, Ss may compare the probe to the main noun of the sentence rather than to the sentence interpretation, even though the latter has already been computed. The first model suggests that there should be no difference between sentences containing regular nouns and sentences containing homophones as long as the $S$ comes up with an interpretation for the sentence before the probe is presented to him. The second model, however, does not exclude such an effect. The examination of this model calls for some further considerations.

There are two ways in which the comparison between the probe and the main noun of the sentence can be executed. First, given a clear disambiguating context, people may process an ambiguous linguistic item just as they would have processed an unambiguous one. In other words, there should be no difference between the processing of regular nouns and that of homophones. The reaction times associated with regular nouns, then, would be equal to the reaction times associated with homophones, as long as the response in both cases is the same. Secondly, people may compute the two interpretations of the ambiguous item. In this case, the reaction times associated with homophones should be greater than those associated with regular nouns, as long as the response in both cases is the same. Furthermore, for homophones one can distinguish between two kinds of irrelevant probes: probes which are irrelevant with respect to either interpretation of the homophone and probes which are irrelevant with respect to the present interpretation of the homophone but which are relevant with respect to its other interpretation. If the search is exhaustive, one should expect no difference between the two kinds of irrelevant probes.
Finally, the interactions between reaction times and completion of processes suggested by Garrett (1970) and Bever et al (1973) may be found in the present task as well. Given enough time between the presentation of the sentence and the presentation of the probe, the odds are that the sentence will have been computed by the time the decision has to be made. In this case, the first strategy (comparison of probe to the computed interpretation) will hold and there will be no difference between sentences which contain a homophone and sentences which do not. On the other hand, if the probe appears shortly after the sentence, one might expect the second strategy (comparison of probe to noun) to hold and the effect of ambiguity to be apparent. In light of this consideration, two experiments were conducted, which differed only in the length of the interval between the two presentations.

\section{METHOD}

Ss were presented with sentences followed by a probe. They were timed as they indicated whether the probe was relevant to the sentence or tot.

The sentences used in the study were all simple affirmative declarative sentences with the subject noun preceding the verb. The subject noun was either a homophone or not. In the former case, the context cleatly disambiguated the meaning of the noun. The probes which followed the sentences were single nouns which were eilher relevant or irrelevant to the sentence. All in all, there were tive combinations of sentence and probe: a sentence without a homophone followed by a relevant probe (regular-yes), a sentence without a homophone followed by an irrelevant probe (regular-no). a sentence with a homophone followed by a relevant probe (homophome-yes), a sentence with a homophone followed by a probe inclevant to the reading dictated by the context, but relevant to the other reading (half-relevant), and a sentence with a homophone followed by a probe which was irrelevant to either reading of the homophone (homophone-no). Examples of the sentences and the probes are presented in Table 1.

At the beginning of each experimental session, relevance was informally defined as "the probe having to do with the topic of the sentence." Then Ss were given at least three examples of sentence and probe combinations with an indication of whether the probe was relevant to the sentence or not. The homophones which appeared in these examples were not used in the experiment itself.

Each $\mathrm{S}$ was presented with a list of 72 pairs of sentences and probes. The list maintained the following distribution: 24 regular-yes, 12 regular-no, 12 homophone-yes, 12 half-relevant, and 12 homophone-no. Evidently, there are three possible categories associated with sentences containing a homophone, but only two categories associated with sentences which do not contain a homophone. In order to keep the same the probability of "yes" and "no" responses as well as that of the occurrence of homophones and $r$ onhomophones, the number of items in the first category was twice

Table 1

Examples for the Different Combinations of Sentences and Probes

\begin{tabular}{lll}
\hline & \multicolumn{1}{c}{ Sentence } & Probe \\
\hline Regular-Yes & The teacher is strict. & Education \\
Homophone-Yes & The organ is healthy. & Body \\
Regular-No & The dog is vicious. & Language \\
Half-Relevant & The pitcher is full. & Baseball \\
Homophone-No & The table is complicated. & Health \\
\hline
\end{tabular}


the number of items in any other category. Prior to the experiment, half the items in the regular-yes category were marked as distractors. These items were not incorporated in the analysis of the data; in other words, in the analysis there was an equal number of responses from each category.

Thirty-six homophones were used in the study. Each homophone appeared with only one possible probe. No list contained both interpretations of a homophone. Thus, six different lists were used in the design. Half the lists contained a set of 36 homophones in one interpretation and half the lists contained the other interpretations of these homophones. For each set of homophones, there were three lists differing in the combination of sentence and probe. Overall, each meaning of each homophone appeared with each of the three types of probes. The same words could be used as relevant, half-relevant. or irrelevant probes, depending on the particular sentence they followed. Within each list, the items of the various categories were, randomly distributed, but the distribution of the categories was the same in all six lists.

All the items were typed in Elite type on $5 \times 8$ in. index cards. The items were shown using an Iconix tachistoscope and exposure box. They were viewed at a distance of $51 \mathrm{~cm}$. On each trial, the $S$ pressed the middle "ready" button of a three-button panel, with either thumb, and thereby triggered a clock accurate to milliseconds. Five hundred milliseconds later, the target sentence appeared for $500 \mathrm{msec}$ and was then followed by the probe. The interval between the onset of the sentence and the onset of the probe was $1.500 \mathrm{misec}$ in Experiment $I$ and $500 \mathrm{msec}$ in Experiment 11. The $S$ indicated his answer by pressing the left or the right button with his left or right index finger. The assignment of button to hand was balanced across Ss with respect to their hand dominance. The $S$ 's response turned off the display and stopped the clock. The recorded reaction times were measured from the onset of the probes. Ss were instructed to respond as fast as possible, not make mistakes, and not guess.

Twelve Stanford students participated in each experiment. All were right-handed native speakers of English who were given credit for their participation. Each experimental session lasted about $45 \mathrm{~min}$, and included a practice session comprising 12 trials. The items presented in these trials were basically no different from the ones used in the experiment itself, except that none of the main nouns which appeared in the former were used in the latter.

\section{RESULTS}

The reaction times in the two experiments were almost identical, in both absolute values and pattern $[F(1,22)=0.004$ for the experiment effect; $F(4,88)=$ 0.36 for the Experiment by Condition interaction]. Therefore, the results of the two experiments were collapsed together and will be discussed accordingly.

The mean reaction time for the homophone-yeses was longer than the mean reaction time for the regular-yeses: 1,711 and $1,551 \mathrm{msec}$, respectively $[\mathrm{F}(1,88)=12.12, \mathrm{p}<.001]$. The mean reaction times for half-relevants and homophone-noes did not differ significantly from one another $[1,821$ and $1,766 \mathrm{msec}$, respectively, $F(1,88)=1.43$, n.s.]. Each of these two values was signiticantly longer than the mean reaction time for regular-noes, which was $1,668 \mathrm{msec}[\mathrm{F}(1,88)$ $=11.08, \mathrm{p}<.001$, and $\mathrm{F}(1.88)=4.54, \mathrm{p}<.05$, respectively].

Errors constituted $1.25 \%$ of the responses. There were significantly more errors associated with homophone-yeses and half-relevants than with the other three categories, which did not differ in their error distribution.

\section{DISCUSSION}

The reaction time data support the exhaustive model for comparison of the main noun and the probe. First, for the same responses, the reaction times associated with homophones were larger than those associated with regular nouns. Secondly, as predicted by the exhaustive model, there was no significant difference between the reaction times associated with the categories half-relevant and homophone-no. One may consider the direction of this difference as supporting the unitary perception hypothesis; however, I think that such a conclusion is not warranted. The difference in question is relatively small and is not significant in either experiment or in the overall analysis. Indeed, it is the only piece of data consistent with the unitary perception hypothesis. Moreover, regarded as an inhibition effect, the difference at hand can be accounted for within the framework of an exhaustive search. More specifically, the conflicting responses (relevant and nonrelevant) associated with the two entries of a homophone in the half-relevant case could lengthen the reaction times to nembers of this category.

The mere fact that ambiguity effects were found favors the suggestion that the probe is compared to the main noun rather than the sentence interpretation. If people were comparing the probe to an already computed sentence interpretation, no differences between homophones and regular nouns would have been expected at all. The pattern of the results being identical in the two experiments may lead one to the same conclusion. The identity suggests that the same processes were operative in both experiments. In turn, this may indicate (i) that in both cases the comparison was with a sentence interpretation because the presentation time in Experiment II was not short enough, or (ii) that in both cases the comparison was with the main noun because the delay in Experiment I was not long enough, or (iii) that there was no interaction between the type of comparison and duration of presentation (i.c.. Ss used a time-independent strategy). From my experience as a pilot $S$ in both experiments, I have to reject the first disjunct. In fact, it seemed surprising that Ss performed the task, let alone with a high degree of accuracy, given what seemed to me to be a very short presentation time. I tend to favor the third disjunct even though I cannot categorically reject the second one.

The error distribution parallels the reaction time data in that the category homophone-yes was associated with more errors than the category regular-yes. However, more errors were associated with the category half-relevant than with the category 
homophone-no. The speed-accuracy tradeoff can be explained in the following way. At times, even though the context dictated one specific reading of the homophone, people came up with the wrong interpretation. As the comparison is between the main noun and the probe, and is furthermore exhaustive, this could not have affected the speed of response. However, the response in such cases is incorrect.

By way of summary, let us follow the processes leading to a response in the present task. Having computed at least a rudimentary interpretation of the sentence, Ss are presented with a probe and they compare it with the main noun. If the latter is a regular noun, the comparison is simple. If the main noun is a homophone, the probe is compared to both entries associated with it. If both comparisons indicate no relevance, the answer is "no." However, if a comparison does indicate a relevant relation, the $S$ has to check whether the relevant interpretation is identical to the interpretation of the main noun in the given context. If it is, the response is "yes," if it is not, the response is "no."

Finally, one may claim that the comparison of probe and main noun, rather than probe and computed interpretation, is counterintuitive. Although a sound claim, it is not a necessary one. Oftentimes we hear a sentence, think we understand it, but indeed we don't, or at least we have a passive attitude towards it. On being tested on the sentence, we find that we have to actively process it again. Alternatively, the comparison of main noun and probe may reflect a task-specific strategy developed during the present experiments.

\section{REFERENCES}

Bever, T. G., Garrett, M. F., \& Hurtig, R. The interaction of perceptual processes and ambiguous sentences. Memory \& Cognition, 1973, 1, 277-286.

Carey, P. W., Mehler, J., \& Bever, T. G. Judging the veracity of ambiguous sentences. Journal of Verbal Leaming \& Verbal Behavior, 1970, 9, 243-254

Fodor, J. A., \& Garrett, M. Some determinants of sentential complexity. Perception \& Psychophysics, 1967, 2, 289-296.

Fodor, J. A., Garrett, M. F., \& Bever, T. G. Some syntactic determinants of sentential complexity. II: Verb structure. Perception \& Psychophysics, 1968, 3, 453-461.

Garrett, M. F. Does ambiguity complicate the perception of sentences? In G. B. Flores d'Arcais and W. J. M. Levelt (Eds.), Advances in psycholinguistics. Amsterdam: North-Holland, 1970.

LACKNER, J. R., \& GARRETT, M. F. Resolving ambiguity: Effects of biasing context in the unattended ear. Cognition, 1972, 2. 359-372.

LASHLEY, K. S. The problem of order in behavior. In L. A. Jeffress, (Ed.), Contral mechanisms in behavior. New York: Wiley, 1951.

Mackay, D. G. To end ambiguous sentences. Perception \& Psychophysics, 1966, 1, 426-436.

(Received for publication May 13, 1974; revision received July 22,1974 .) 